\title{
Impairment of diastolic function by shortened filling period in severe left ventricular disease
}

\author{
KELVIN S K NG, DEREK G GIBSON \\ From the Cardiac Department, Brompton Hospital, London
}

SUMMARY A dilated left ventricle with reduced ejection fraction is usually attributed to impaired systolic function. To investigate the possibility that ventricular filling might also be disturbed, $M$ mode echocardiograms, phonocardiograms, and Doppler cardiograms were recorded in 30 patients with ventricular disease of varying cause. All but four had functional mitral regurgitation. The size of the left ventricular cavity was increased in all and peak velocity of circumferential fibre shortening was reduced. Diastolic abnormalities included a short isovolumic relaxation time, and, on digitised $\mathrm{M}$ mode, a reduced rate of dimension increase and of posterior wall thinning. Although the timing of aortic valve closure was normal, mitral regurgitation persisted beyond it by 95 (35) $\mathrm{ms}$ and beyond mitral valve opening by $60(40) \mathrm{ms}$. This reduced the effective filling time (the interval when the mitral valve was open and mitral regurgitation was absent) to $<200 \mathrm{~ms}$ in seven patients. The effective filling time correlated closely with the $R R$ interval, the regression equation indicating a reduction of $80 \mathrm{~ms}$ for each $100 \mathrm{~ms}$ fall in $R R$ interval. It was also independently shortened by $2 \mathrm{~ms}$ a year with increasing age. The effective left ventricular filling time may thus be very short in patients with left ventricular cavity dilatation and functional mitral regurgitation.

It is suggested that when diastolic function is also abnormal, this short filling time may physically limit ventricular inflow. Its close relation to heart rate might contribute to the therapeutic effect of $\beta$ blockade in such patients.

A dilated left ventricular cavity with reduced ejection fraction is common in the late stages of heart disease, and is usually held to be caused by depressed systolic function. ${ }^{1}$ Such patients are often said to be in "heart failure" and are treated with digitalis, diuretics, and vasodilators. A minority, however, respond favourably to $\beta$ adrenoceptor blocking drugs ${ }^{2}$ and this would be surprising if systolic disease were the only reason why function was impaired. It is possible, therefore, that other pathogenic mechanisms are involved, and we have attempted to identify them by investigating how filling, rather than ejection, can be disturbed in these patients.

\section{Patients and methods}

The records of 30 unselected patients (aged from 12 to 75,22 men) with dilatation of the left ventricular

Requests for reprints to Dr Derek G Gibson, Brompton Hospital, Fulham Road, London SW3 6HP.

Accepted for publication 18 April 1989 cavity and reduced shortening fraction were examined retrospectively. The underlying diagnosis was coronary artery disease in 11 , dilated cardiomyopathy in three, presumed myocarditis in two, and there were single cases associated with polyarteritis nodosa, Churg-Strauss syndrome, sarcoidosis, irradiation, and alcoholic cirrhosis. Coronary artery disease was diagnosed by coronary arteriography or directly at necropsy or at the time of cardiac transplantation. In the remaining nine patients, three of whom had long standing hypertension, we cannot say whether or not coronary artery disease was present, because there was no clinical indication for coronary arteriography. All the patients were in sinus rhythm and five had left bundle branch block. All were taking diuretics, either frusemide or bumetanide, along with amiloride in nine or metolazone in one. Twenty were taking captopril, three a theophylline preparation, two isosorbide, and one nifedipine, while five were treated with a digitalis preparation.

Cross sectional echocardiography in multiple cuts 
showed uniform enlargement of the left ventricular cavity and a reduced ejection fraction in all, and excluded a ventricular aneurysm or other major abnormality of regional wall motion. $M$ mode echocardiograms were recorded with simultaneous phonocardiograms and electrocardiograms at a paper speed of $100 \mathrm{~mm} / \mathrm{s}$ at the level of the tips of the mitral leaflets. Clear, continuous echoes were obtained from the endocardial surfaces of the left side of the septum and the endocardium of the posterior wall. Separate echograms were recorded of the mitral valve at the level at which cusp separation at the start of filling and closure at the onset of systole could most clearly be seen (fig la) and of the aortic echogram, from which the aortic component of the second heart sound $\left(A_{2}\right)$ was identified as the onset of the first high frequency vibration of the component coinciding with cusp apposition. Continuous wave Doppler recordings were made with Doptek equipment, again with simultaneous phonocardiograms and electrocardiograms, at a paper speed of $100 \mathrm{~mm} / \mathrm{s}$ to show the timing of functional mitral regurgitation (present in all but four patients), and separately, of early diastolic forward flow across the mitral valve.

From the $M$ mode echocardiograms we measured the dimensions of the left ventricular cavity synchronously with the $Q$ wave of the electrocardiogram (end diastolic) and with $A_{2}$ (end ejection), from the leading edges. We digitised the records ${ }^{3}$ to give plots of the left ventricular dimension and posterior wall thickness along with their rates of change. The timing of $A_{2}$ was superimposed on all digitised records, and the interval $A_{2}$ to peak rate of dimension increase was measured. We took the isovolumic relaxation time to be the interval between the onset of the first high frequency component of $\mathrm{A}_{2}$ on the phonocardiogram and the initial cusp separation on the mitral echogram. ${ }^{4}$ We estimated the interval over which the mitral valve was open (mitral valve open time) $)^{5}$ as that from the initial cusp separation at the onset of diastole to the final apposition at the start of the succeeding systole. The left ventricular ejection time was determined from the aortic echograms and the $\mathrm{QA}_{2}$ interval from the electrocardiogram and phonocardiogram. Values of $\mathrm{QA}_{2}$ from patients with left bundle branch block were not included in the analysis.

On the Doppler recording, we measured the overall duration of the mitral regurgitant signal (fig 1b) and also the two intervals from $Q$ to the onset of the regurgitant signal and from $A_{2}$ to its end. On the trace of forward mitral flow we measured the interval from $A_{2}$ to the time of peak flow velocity.

Preliminary examination of the records showed that the times of onset and end of mitral regurgitation bore no fixed relation to those of mitral opening or

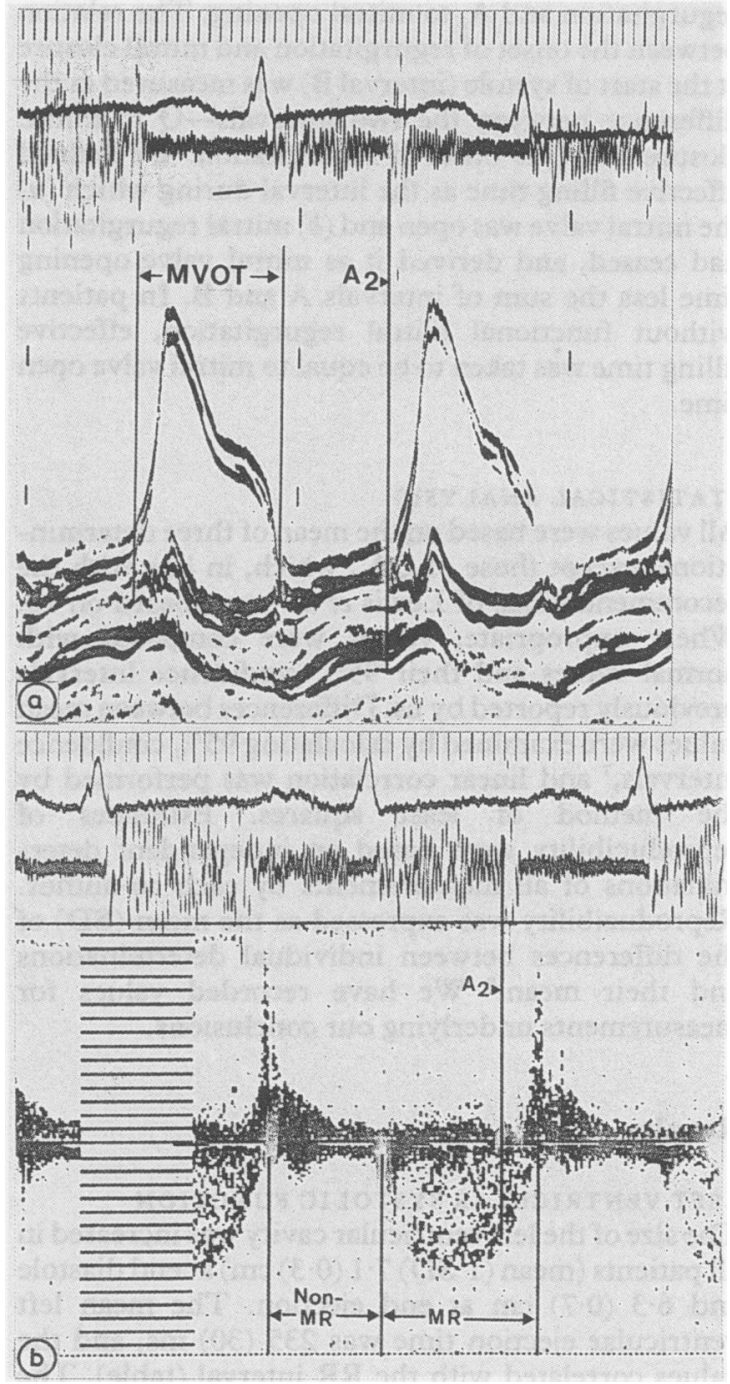

Fig 1 (a) Mitral echogram from a patient with cardiomyopathy. Mitral valve open time is indicated (MVOT). Note that mitral cusp separation at the start of diastole is synchronous with aortic valve closure $\left(A_{2}\right)$. (b) Continuous wave Doppler recording from the same patient, showing mitral regurgitation (MR). Its duration and that of the interval between pulses of regurgitation (non-MR) are indicated. Note that regurgitation continues for approximately $70 \mathrm{~ms}$ beyond $A_{2}$. (Full scale deflection $16 \mathrm{kHz}$ ).

closure, the regurgitation signal sometimes starting before mitral closure at the start of systole and often persisting beyond mitral opening at its end (fig 1). The interval from mitral opening to the end of regurgitation (interval $\mathbf{A}$ ) was thus measured as the difference between the intervals $A_{2}$ to the end of 
regurgitation and $A_{2}$ to mitral opening. The relation between the onset of regurgitation and mitral closure at the start of systole (interval B) was measured as the difference between the two intervals- $Q$ to mitral closure and $Q$ to onset of regurgitation. We defined effective filling time as the interval during which $(a)$ the mitral valve was open and $(b)$ mitral regurgitation had ceased, and derived it as mitral valve opening time less the sum of intervals $A$ and $B$. In patients without functional mitral regurgitation, effective filling time was taken to be equal to mitral valve open time.

\section{STATISTICAL ANALYSIS}

All values were based on the mean of three determinations, except those of $\mathrm{QA}_{2}$, which, in line with the recommendations of Lewis et $a l^{6}$ were based on 10 . Where appropriate, values were compared with normal values and their $95 \%$ confidence intervals previously reported by us. Differences between mean values were examined by calculating $95 \%$ confidence intervals, ${ }^{7}$ and linear correlation was performed by the method of least squares. Estimates of reproducibility were based on independent determinations of all measurements by each co-author. Reproducibility was expressed as the mean (SD) of the differences between individual determinations and their mean. ${ }^{8}$ We have recorded values for measurements underlying our conclusions.

\section{Results}

\section{LEFT VENTRICULAR SYSTOLIC FUNCTION}

The size of the left ventricular cavity was increased in all patients (mean (1 SD) $7 \cdot 1(0 \cdot 3) \mathrm{cm}$ ) at end diastole and $6.3(0.7) \mathrm{cm}$ at end ejection. The mean left ventricular ejection time was $235(30) \mathrm{ms}$, and the values correlated with the $R R$ interval (table). The mean value of the $\mathrm{QA}_{2}$ intervals was $375(50) \mathrm{ms}$, with a reproducibility of $0(8) \mathrm{ms}$, and again this correlated with the RR interval (table) but not with age. The corresponding regression relation with heart rate was:

$$
\mathrm{QA}_{2}=510-1.65 \text { (heart rate) ms, }
$$

the standard errors of the estimate was $30 \mathrm{~ms}$, and those of the slope and intercept were 0.36 and 35 respectively. The slope and intercept of this equation did not differ significantly from normal values previously reported by Lewis et al. ${ }^{6}$ The mean value of peak velocity of circumferential fibre shortening (VCF) was $0.6(0.2) / \mathrm{s}$. In all the patients, it was below the normal range of $1 \cdot 2-2 \cdot 0 / \mathrm{s}$.

\section{MITRAL VALVE MOTION}

The isovolumic relaxation time was 25 (35) ms (reproducibility 1 (7) $\mathrm{ms}$ ), significantly less than the normal value of $60(10) \mathrm{ms}$; only 11 patients were within the normal range, values being abnormally short in the remainder. The mean mitral valve open time was 345 (130) ms (reproducibility $-3(10)$ ) and values correlated strongly with heart rate (table). Mitral closure followed the $Q$ wave by $75(55) \mathrm{ms}$, an interval unrelated to heart rate.

\section{DOPPLER MEASUREMENTS}

Mitral regurgitation was detected by continuous wave Doppler in $\mathbf{2 6}$ of the $\mathbf{3 0}$ patients. It lasted $\mathbf{4 0 0}$ (70) $\mathrm{ms}$, reproducibility -3 (10), with values above $500 \mathrm{~ms}$ in three patients. It correlated weakly with RR interval and rather more strongly with age (table). The interval between the pulses of regurgitation (non-MR interval) was 290 (120) ms (reproducibility $3(10) \mathrm{ms}$ ) which correlated strongly with $R R$ interval (table). The mean interval between the $Q$ wave and the onset of mitral regurgitation was 65 (20) ms, and between $A_{2}$ and its end it was 95 (35) $\mathrm{ms}$, (reproducibility 0 (7) $\mathrm{ms}$ ). Neither was related to heart rate, though the time from $\mathrm{A}_{2}$ to the end of mitral regurgitation increased with age (table).

\section{EFFECTIVE FILLING PERIOD}

In all but three patients mitral regurgitation persisted for up to $160 \mathrm{~ms}$ after mitral valve opening (mean

Table Determinants of ventricular function

\begin{tabular}{|c|c|c|c|c|c|c|c|}
\hline \multirow[b]{2}{*}{ Dependent variable $(x)$} & \multirow[b]{2}{*}{ Independent variable (y) } & \multirow[b]{2}{*}{$\boldsymbol{r}$} & \multirow[b]{2}{*}{ Slope } & \multirow[b]{2}{*}{ Intercept } & \multicolumn{3}{|c|}{ Standard error } \\
\hline & & & & & Slope & Intercept & Estimate \\
\hline $\begin{array}{l}\text { Duration of } M R \\
A_{2} \text { to end of } M R \\
\text { Non-MR interval } \\
\text { Mitral valve open time } \\
\text { Non-MR interval } \\
\text { Effective filling time } \\
Q_{2} \text { interval } \\
\text { Ejection time } \\
\text { Duration of } M R\end{array}$ & $\begin{array}{l}\text { Age } \\
\text { Age } \\
\text { Mitral valve open time } \\
\text { RR interval } \\
\text { RR interval } \\
\text { RR interval } \\
\text { RR interval } \\
\text { RR interval } \\
\text { RR interval }\end{array}$ & $\begin{array}{l}0.53 \\
0.68 \\
0.87 \\
0.78 \\
0.87 \\
0.80 \\
0.67 \\
0.50 \\
0.43\end{array}$ & $\begin{array}{l}2 \cdot 0 \\
1.3 \\
1 \cdot 0 \\
0.82 \\
0.75 \\
0.78 \\
0 \cdot 23 \\
0 \cdot 11 \\
0.21\end{array}$ & $\begin{array}{r}290 \\
18 \\
69 \\
-220 \\
-230 \\
-260 \\
205 \\
160 \\
250\end{array}$ & $\begin{array}{l}0.68 \\
0.30 \\
0.11 \\
0.12 \\
0.09 \\
0.12 \\
0.05 \\
0.04 \\
0.09\end{array}$ & $\begin{array}{l}38 \\
17 \\
36 \\
88 \\
65 \\
83 \\
34 \\
26 \\
66\end{array}$ & $\begin{array}{l}54 \\
24 \\
68 \\
90 \\
63 \\
84 \\
37 \\
28 \\
66\end{array}$ \\
\hline
\end{tabular}

MR, mitral regurgitation. 


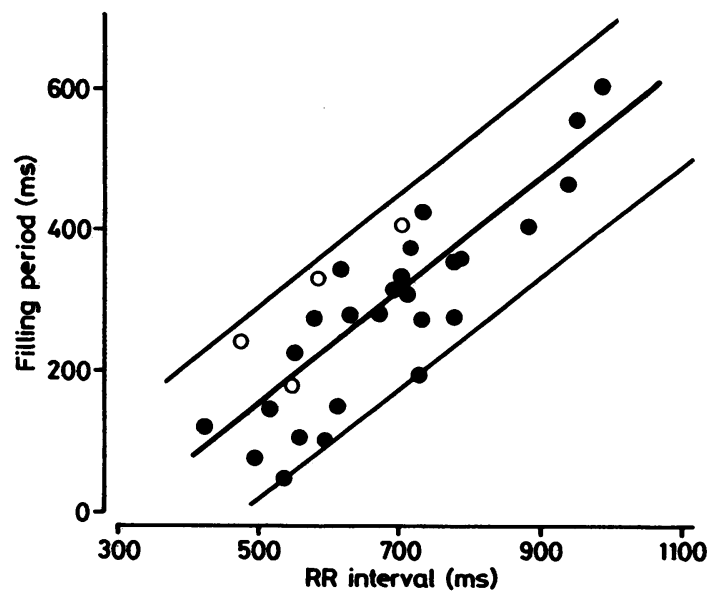

Fig 2 Relation between $R R$ interval and effective filling period. Limits are set at two standard errors of the estimate. Closed symbols represent results from patients with mitral regurgitation and open symbols from those without.

$60(40) \mathrm{ms}$ ). By contrast, the onset of mitral regurgitation could precede or follow mitral closure, the mean interval between the two being $8(40) \mathrm{ms}$. The mean value of effective filling period was thus 275 (135) $\mathrm{ms}$, reproducibility 6 (17) $\mathrm{ms}$, which again correlated strongly with the RR interval (table and fig 2). In seven patients the effective filling period was $<200 \mathrm{~ms}$. Their average age $(45(15))$ was no different from that of the whole group. They all had functional mitral regurgitation. Two of them had myocarditis, one each had heart disease caused by coronary artery disease, irradiation, or polyarteritis nodosa, while a definite diagnosis had not been established in two. Two had left bundle branch block. In six, the RR interval was in the range 480 $590 \mathrm{~ms}$; in the seventh, it was $740 \mathrm{~ms}$, and in this patient the effective filling period was short because mitral regurgitation lasted $560 \mathrm{~ms}$, extending $160 \mathrm{~ms}$ beyond $\mathrm{A}_{2}$.

\section{DIGITISED M MODE ECHOCARDIOGRAMS}

On the digitised $M$ mode echocardiograms, the peak rate of dimension increase was $7.5(3.8) \mathrm{cm} / \mathrm{s}$. Seven values were within the normal range and the remainder were below. The rapid filling period was 150 (45) $\mathrm{ms}$, with values below the normal range in 11 and above it in one. The peak rate of wall thinning was very low $(3.5(2) \mathrm{cm} / \mathrm{s})$ and values from all but four patients were below the lower limit of normal. The interval from minimum cavity dimension to mitral opening was $3(40) \mathrm{ms}$, which was not significantly different from normal. There was wide variation between patients, however: mitral opening was abnormally delayed in seven, and was premature, occurring up to $80 \mathrm{~ms}$ before minimum dimension, in six. Finally, phase relations between the peak rate of dimension increase and peak flow were abnormal during rapid filling, when the normal delay in peak filling velocity with respect to peak rate of dimension increase $(50(15) \mathrm{ms})$ was lost in 10 patients.

\section{WITHIN PATIENT COMPARISONS}

We recorded at two different heart rates in eight patients. With a mean fall in RR interval of 155 (110) $\mathrm{ms}$, the non-MR interval shortened by 105 (130) (95\% confidence interval $45 \mathrm{~ms})$ and mitral valve open time by 105 (90) $(95 \%$ confidence interval $60 \mathrm{~ms}$ ), while isovolumic relaxation time, and the duration of mitral regurgitation and its persistence beyond $A_{2}$ were all unchanged.

\section{Discussion}

Although impaired systolic function is usually held to be the main cause of the dilated left ventricular cavity and reduced ejection fraction seen in patients with severe heart disease, ${ }^{1}$ diastolic abnormalities have also been described. The peak rate of pressure decline during isovolumic relaxation was reduced $^{9}$ and the time constant of pressure fall was prolonged. ${ }^{10}$ Although the peak rate of increase of ventricular volume during early diastole was usually normal, ${ }^{11}$ the peak rate of dimension increase was often low, and in many patients the early rapid filling period was short. ${ }^{912}$ Finally, left ventricular passive stiffness was increased, both when expressed as cavity compliance or as a myocardial stress-strain relation. ${ }^{13}$ These abnormal measurements, however, do not necessarily define independent disturbances of diastole. When left ventricular filling pressure is raised, as it often has been in reported cases, isovolumic relaxation time may be very short or even zero, ${ }^{4}$ so that the fundamental assumption underlying the use of the time constant of pressure fall as a measure of relaxation, namely that the ventricle is isovolumic for the period over which the calculation is made, does not necessarily apply. At a constant transmitral flow rate, the peak rate of dimension increase is inversely proportional to the square of the dimension, so on simple geometrical grounds it will be low when the ventricle is enlarged. "Normalisation" to unit dimension by calculating the velocity of circumferential fibre shortening or lengthening merely increases the discrepancy, making the peak rates of dimension increase sensitive to the cube of the dimension. Finally passive stiffness can only be calculated when pressure and volume increase together. This does not occur until late diastole when heart rate is low $^{14}$ and may not do so at all when it is rapid. 
In our study, therefore, we examined the effective left ventricular filling period, an aspect of diastolic function that is independent of increased cavity size, heart rate, or isovolumic relaxation time. We defined it as the period during which the mitral valve was open and retrograde flow had ceased, assuming that there is no forward flow from atrium to ventricle in the presence of mitral regurgitation. In several patients, this effective filling period was very short, and in seven, it was $<200 \mathrm{~ms}$. In healthy individuals, left ventricular filling time varies with heart rate. It shortens with tachycardia caused by exercise or atrial pacing, reaching a minimum of $100 \mathrm{~ms} .{ }^{5}$ Ventricular filling may be very rapid, therefore, when diastolic function is normal. In patients with left ventricular hypertrophy and associated diastolic disease, filling time does not drop below $200 \mathrm{~ms}$, even when heart rate is high; the extra filling time is found within the $R R$ interval by shortening isovolumic relaxation time. Diastolic abnormalities in our patients differed from those seen in patients with hypertrophy. The isovolumic relaxation time was usually very short or even zero at rest rather than being increased, and the early diastolic filling period was either normal or short, rather than prolonged. Nevertheless, phase relations between transmitral flow velocity and dimension changes were often abnormal. Rapid wall thinning, intimately related to rapid ventricular filling under normal conditions, ${ }^{15}$ was much lower than would have been expected from cavity dilatation alone. Mitral valve opening was often delayed in relation to minimum cavity dimension, indicating incoordinate relaxation. ${ }^{16}$ Finally, changes in cavity shape, which also form a prominent part of normal ventricular adaptation to rapid filling, are likely to have been absent in our patients. ${ }^{17}$ Superimposed on such abnormal diastolic function, therefore, a filling period of $<200 \mathrm{~ms}$ might be expected to limit ventricular inflow directly.

A striking feature of the functional mitral regurgitation in our patients was that it lasted $\geqslant 500 \mathrm{~ms}$. Though the $\mathrm{QA}_{2}$ interval was normal, retrograde flow was prolonged beyond the end of ejection into what would normally have been isovolumic relaxation. In our patients, however, isovolumic relaxation was usually short or even zero. This led to the paradoxical finding in most that the mitral valve was open in the face of continuing regurgitation (fig 1 ). In addition, the excessive duration of the regurgitation was little affected by heart rate. Filling time thus became very sensitive to tachycardia, shortening by $80 \mathrm{~ms}$ for each $100 \mathrm{~ms}$ fall in RR interval. As heart rate rises, therefore, the cardiac cycle becomes increasingly occupied by mitral regurgitation, and filling time becomes disproportionately shortened (fig 2). This effect of heart rate was seen whatever the cause of the ventricular disease, pointing to the dilated cavity itself as a possible cause. The effect of age on the duration of mitral regurgitation and its continuation beyond $A_{2}$ suggests that filling might be more frequently disturbed in older patients, but we found no evidence of this. Presumably the duration of mitral regurgitation reflects the period during which myocardial tension develops in the left ventricle, though we do not know why this is so strikingly prolonged. Perhaps the time course of deactivation ${ }^{18}$ is modified by the abnormal pattern of development of systolic stress in a dilated ventricle or by the loss of $\beta$ adrenoceptive receptors found in "failing" human hearts removed at the time of transplantation. ${ }^{19}$ Certainly, our results accord with the disturbed calcium handling described in vitro in myocardium from patients with severe ventricular disease..$^{20}$

In some of our patients, therefore, cardiac output seemed to have been limited not directly by systolic disease but by ventricular inflow. When filling time is short, mean flow rates across the mitral valve must be rapid to maintain stroke volume, and this means that acceleration and deceleration rates must be correspondingly high. Such rapid changes in blood velocity must, on a simple physical basis, be accompanied by considerable pressure drops along the direction of flow. This leads to conditions closely resembling those seen in rheumatic mitral stenosis, when ventricular filling also depends on a high pressure difference between left atrium and left ventricle. In both conditions cardiac output may be low and arterial pressure maintained only by raised peripheral resistance, effectively increasing ventricular afterload. This may explain the reduced ejection rates seen in mitral stenosis and the tendency for cavity size and filling pressures to fall after successful operation. ${ }^{21}$ Sometimes these changes may be very striking, with a remarkable increase in ejection fraction and fall in end diastolic pressure. ${ }^{22}$ If ventricular function was modified in the same way in our patients, the low observed ejection fractions might have been caused in part by this secondary increase in resistance to ejection and not exclusively by left ventricular disease. These similarities could be summarised by suggesting that our patients had "temporal" mitral stenosis to emphasise how much they resemble those of the well recognised "spatial" variety.

The filling period was not short in all our patients. The heart rate was low in some and regurgitation not unduly prolonged in others. It would not have been possible to predict these differences in haemodynamic state from simple measurements of heart rate or cavity size. Yet they are not purely of theoretical importance because they suggest approaches to treatment. If the filling period does 


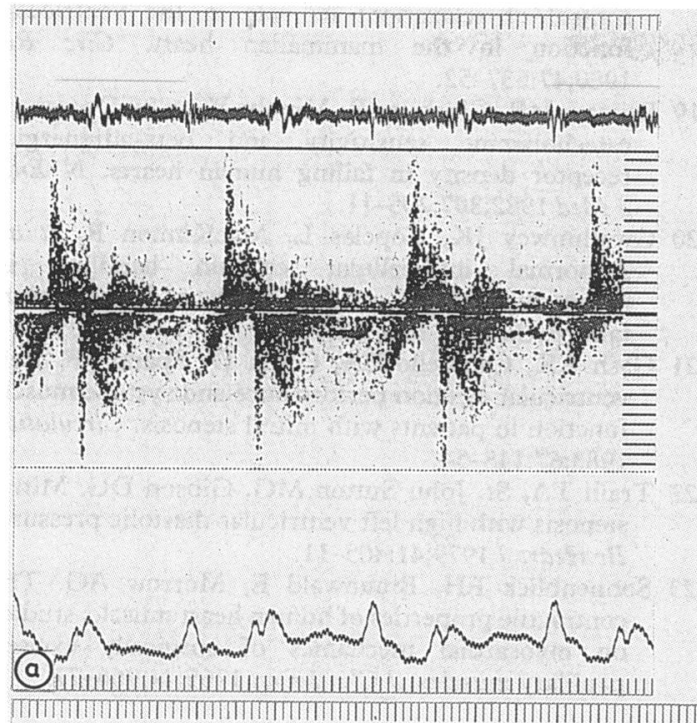

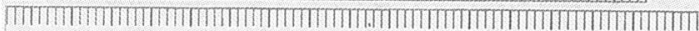

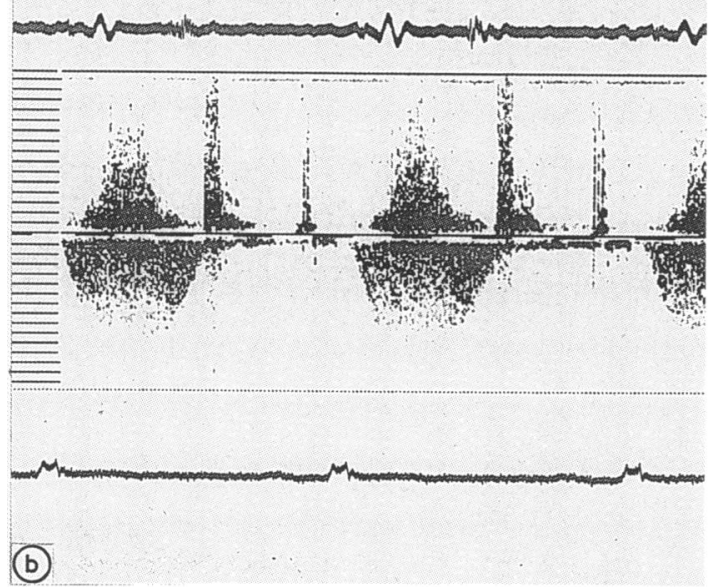

Fig 3 (a) Continuous wave Doppler trace from a patient with sinus tachycardia, showing non-MR interval reduced to approximately $80 \mathrm{~ms}$. (b) Continuous wave Doppler trace from the same patient while taking oral metoprolol. Heart rate has slowed and non-MR interval has increased to $400 \mathrm{~ms}$. (Full scale deflection $=16 \mathrm{kHz}$ ).

indeed limit cardiac output, it would be reasonable to treat it. One way would be to reduce the heart rate; such an action might explain the favourable response to $\beta$ adrenoceptive blocking drugs seen in a minority of patients with dilated cardiomyopathy. ${ }^{2}$ Figure 3 shows an example of such a patient who greatly improved with metroprolol as the filling time increased by a factor of more than three. The action of positive inotropic drugs in increasing the peak force of myocardial contraction and its peak rate of development is well recognised, ${ }^{23}$ but their additional effect of shortening the overall duration of systole might be particularly valuable when filling time is limited and could perhaps be enhanced by suitable treatment design. A drug, such as digitalis, with both effects might thus have special advantages. Finally, the unexpected relation between retrograde mitral flow and motion of the valve cusps needs more study because the erosion of filling time by regurgitation that persists after mitral opening might also be manipulated by drugs.

The patients we have described all had a dilated cavity and a reduced ejection fraction; all might therefore have been diagnosed as having "heart failure" and a uniform treatment prescribed. However, the position of the rate limiting step in the circulation varied even in this small group. We believe that individual treatment on the basis of individual analysis of the underlying disturbance to the circulation may be a more fruitful approach to the management of the common but intractable clinical problem of advanced ventricular disease.

\section{References}

1 Braunwald E, Ross J Jr, Sonnenblick EH. Mechanisms of contraction in the normal and failing heart. $N$ Engl $J$ Med 1967;277:1012-22.

2 Swedberg K, Hjalmarson $\AA$, Waagstein F, Wallentin I. Beneficial effects of long term beta-blockade in congestive cardiomyopathy. Br Heart $J$ 1980;44:117-33.

3 Gibson DG, Brown D. Measurement of instantaneous left ventricular dimension and filling rate in man, using echocardiography. Br Heart $J$ 1973;35:1141-9.

4 Mattheos M, Shapiro E, Oldershaw PJ, Sacchetti R, Gibson DG. Non-invasive assessment of changes in left ventricular relaxation by combined phono-, echoand mechanography. Br Heart $J$ 1982;47:253-60.

5 Oldershaw PJ, Dawkins KD, Ward DE, Gibson DG. Diastolic mechanisms of impaired exercise tolerance in aortic valve disease. Br Heart $J$ 1983;49:568-73.

6 Lewis RP, Boudalas H, Forester WF, Weissler AM. A critical review of systolic time intervals. Circulation 1977;56:146-58.

7 Evans SJW, Mills P, Dawson J. The end of the p value? Br Heart J 1988;60:177-80.

8 Bland JM, Altman DG. Statistical methods for assessing agreement between two methods of clinical measurement. Lancet 1986;i:307-10.

9 Grossman W, McLaurin LP, Rolett EL. Alterations in left ventricular relaxation and diastolic compliance in congestive cardiomyopathy. Cardiovasc Res 1979; 13:514-22.

10 Carroll JD, Lang RM, Neumann AL, Borow KM, Rajfer SI. The differential effect of positive inotropic and vasodilator therapy on diastolic properties in patients with congestive cardiomyopathy. Circulation 1986;74:815-25.

11 Rahko PS, Shaver JA, Salerni R, Uretsky BF. Digital 
echocardiographic analysis of left ventricular filling in dilated cardiomyopathy. Am $J$ Noninvas Cardiol 1988;2:10-8.

12 Hammermeister KE, Warbasse JR. The rate of change of left ventricular volume in man. II Diastolic events in health and disease. Circulation 1974;49:739-47.

13 Gibson DG. Assessment of ventricular diastolic function. In: Parmley WW, Chatterjee K, eds. Cardiology. Lippincott: Philadelphia, 1987:53:1-17.

14 Fioretti P, Brower RW, Meester GT, Serruys PW. Interaction of left ventricular relaxation and filling during early diastole in human subjects. Am J Cardiol 1980;46:197-203.

15 Hui WKK, Gibson DG. The dynamics of rapid left ventricular filling. Adv Cardiol 1985;32:7-35.

16 Gibson DG, Prewitt TA, Brown DJ. Analysis of left ventricular wall movement during isovolumic relaxation and its relation to coronary artery disease. $\mathrm{Br}$ Heart $J$ 1976;38:1010-9.

17 Gibson DG, Brown DJ. Continuous assessment of left ventricular shape in man. Br Heart J 1975;37:904-10.

18 Brutsaert DL, Housmans PR, Goethals MA. Dual control of relaxation: its role in the ventricular function in the mammalian heart. Circ Res 1980;47:637-52.

19 Bristow MR, Ginsburg R, Minobe W, et al. Decreased catecholamine sensitivity and beta-adrenergicreceptor density in failing human hearts. $N$ Engl J Med 1982;307:205-11.

20 Gwathmwey JK, Copelas L, MacKinnon R, et al. Abnormal intracellular calcium handling in myocardium from patients with end-stage heart failure. Circ Res 1987;61:70-6.

21 Gash AK, Carabello BA, Cepin D, Spann JF. Left ventricular ejection performance and systolic muscle function in patients with mitral stenosis. Circulation 1983;67:148-54.

22 Traill TA, St. John Sutton MG, Gibson DG. Mitral stenosis with high left ventricular diastolic pressure. Br Heart J 1979;41:405-11.

23 Sonnenblick EH, Braunwald E, Morrow AG. The contractile properties of human heart muscle: studies on myocardial mechanics of surgically excised papillary muscles. J Clin Invest 1965;44:966-77. 